\title{
Erratum: Towards a Unified Description of the Rheology of Hard-Particle Suspensions [Phys. Rev. Lett. 115, 088304 (2015)]
}

\author{
B. M. Guy, M. Hermes, and W. C. K. Poon
}

(Received 22 December 2015; published 2 February 2016)

DOI: 10.1103/PhysRevLett.116.059901

The graph of onset stress $\sigma^{*}$ versus particle diameter $d$, Fig. 2 in the original Letter, contains a set of literature measurements that has been inadvertently plotted twice, once as Ref. [15] [P. D'Haene, J. Mewis, and G. G. Fuller, J. Colloid Interface Sci. 156, 350 (1993)] and again as Ref. [16] [L. N. Krishnamurthy, N. J. Wagner, and J. Mewis, J. Rheol. 49, 1347 (2005)]. We have removed the second instance of the data and the revised figure is shown in Fig. 1, below.

The correct values of the fitting parameters for the least-squares fit are $\alpha=-1.9(2)$ and $B=3 \times 10^{-11} \mathrm{~J} \mathrm{~m}^{-1.1}$. The caption of Fig. 2 in the original Letter should, therefore, read as follows: "[...] The solid line is a least-squares fit to a power

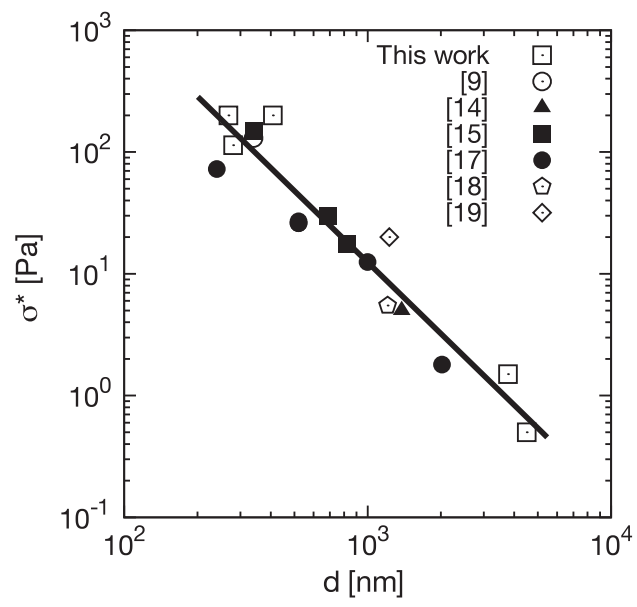

FIG. 1. Onset stress $\sigma^{*}$ versus particle diameter $d$ for PHSA-stabilized PMMA spheres in this and previous $[9,14,15,17-19]$ work. The solid line is a least-squares fit to a power law $\sigma^{*}=B d^{\alpha}$, with $B=3 \times 10^{-11} \mathrm{~J} \mathrm{~m}^{-1.1}$ and $\alpha=-1.9(2)$.

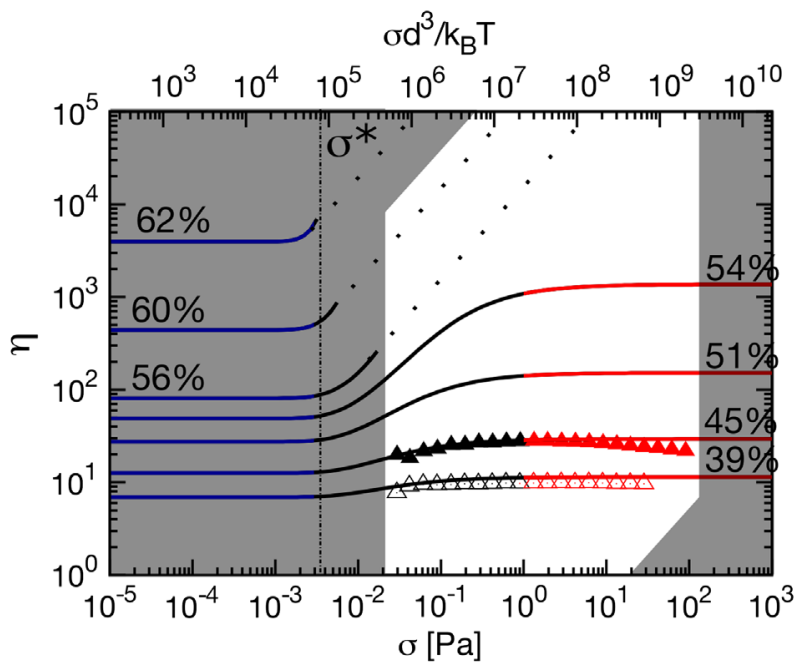

FIG. 2. Rheology in the granular size regime. The figure caption is as in the original Letter. 
law $\sigma^{*}=B d^{\alpha}$, with $B=3 \times 10^{-11} \mathrm{~J} \mathrm{~m}^{-1.1}$ and $\alpha=-1.9(2)$. ."; the penultimate paragraph on page 088304-3 should read "We find, Fig. 2, that $\sigma^{*}$ decreases with particle size as $\sigma^{*} \simeq B d^{\alpha}$ with $B=3 \times 10^{-11} \mathrm{~J} \mathrm{~m}^{-1.1}$ and $\alpha=-1.9(2) . "$; and the final paragraph on page 088304-3 should read "[...] a constant force $f^{*}$ is needed to push particles into frictional contact: $\sigma^{*} \simeq f^{*} / d^{2}$ with $f^{*}=3 k_{B} T / \mathrm{nm}$."

There is an error in the position of the vertical line marking the onset stress $\sigma^{*}$ in Fig. 1(d) in the original Letter. A corrected version of the figure is shown in Fig. 2, below. The expression for the high-viscosity branch $\eta_{2}(\phi)$ in the caption of Fig. 1 in the original Letter is also incorrect, and should be $\eta_{2}(\phi)=A\left(\phi_{m}-\phi\right)^{-n}$. These corrections do not change any of the conclusions of the Letter. 